OPEN

SUBJECT AREAS:

CRYOELECTRON

TOMOGRAPHY

BACTERIOLOGY

Received

23 September 2013

Accepted

13 November 2013

Published

28 November 2013

Correspondence and requests for materials should be addressed to

K.N. (keiichi@fbs.

osaka-u.ac.jp)

\section{Common and distinct structural features of Salmonella injectisome and flagellar basal body}

\author{
Akihiro Kawamoto 1,2, Yusuke V. Morimoto ',2, Tomoko Miyata', Tohru Minamino', Kelly T. Hughes ${ }^{3}$, \\ Takayuki Kato' \& Keiichi Namba ${ }^{1,2}$
}

${ }^{1}$ Graduate School of Frontier Biosciences, Osaka University, 1-3 Yamadaoka, Suita, Osaka 565-0871, Japan, ${ }^{2}$ Riken Quantitative Biology Center, 1-3 Yamadaoka, Suita, Osaka 565-0871, Japan, ${ }^{3}$ Department of Biology, University of Utah, Salt Lake City, UT, USA.

Bacterial pathogens use an injectisome to deliver virulence proteins into eukaryotic host cells. The bacterial flagellum and injectisome export their component proteins for self-assembly. These two systems show high structural similarities and are classified as the type III secretion system, but it remains elusive how similar they are in situ because the structures of these complexes isolated from cells and visualized by electron cryomicroscopy have shown only the export channel and housing for the export apparatus. Here we report in situ structures of Salmonella injectisome and flagellum by electron cryotomography. The injectisome lacks the flagellar basal body C-ring, but a wing-like disc and a globular density corresponding to the export gate platform and ATPase hexamer ring, respectively, are stably attached through thin connectors, revealing yet unidentified common architectures of the two systems. The ATPase ring is far from the disc, suggesting that both apparatuses are observed in an export-off state.

athogenic bacteria use the injectisome, also called the needle complex (NC), to directly inject virulence effector proteins into eukaryotic host cells upon contact, thereby causing rearrangement of the host cytoskeletons to allow their invasion into the host cells ${ }^{1,2}$. The NC structure is made of an extracellular thin needle tube and a basal body consisting of multiple rings that span the inner and outer membranes. The bacterial flagellum is an organelle for motility and is made of an extracellular, long helical filament as a propeller and a basal body as a rotary motor. The flagellar basal body (FBB) is also made of multiple rings that span the inner and outer membranes ${ }^{3}$. The injectisome and bacterial flagellum are both large assemblies of 20 to 30 different proteins and export their component proteins for self-assembly at the distal, growing ends. Their basal bodies function as specific protein export apparatuses classified as the type III secretion system (T3SS) ${ }^{1,3}$. The T3SS of pathogens is a potentially excellent target for anti-infection drug design because such drugs do not affect bacterial survival and therefore resistant mutations would occur much less frequently ${ }^{1,2}$. NC and FBB isolated from bacterial cells by detergent treatment show highly similar architecture ${ }^{4-6}$ and their component proteins are highly homologus ${ }^{1,2}$, suggesting that $\mathrm{NC}$ is evolutionally related to the flagella (Fig. 1).

Both T3SS consists of a membrane-embedded export gate and a cytoplasmic complex including an ATPase and are composed of multiple, highly conserved proteins (Supplementary Table 1). In Salmonella enterica serovar Typhimurium, the NC export gate is made of five membrane proteins, SpaP, SpaQ, SpaR, SpaS and InvA, and InvC ATPase forms a homo-hexameric ring with OrgA, OrgB and InvI on the cytoplasmic side. These components are all required not only for effector protein secretion but also for needle tube formation ${ }^{1,2}$. The export gate components are located in the central portion of the inner membrane ring (IR) complexes ${ }^{7}$. SpaO forms a sorting platform in the cytoplasm to facilitate sequential export of secretory proteins by providing specific binding sites for OrgA and OrgB to bring in the InvC-chaperone-secretory protein complexes to the vicinity of the export gate ${ }^{8}$. InvC ATPase induces chaperone release from and unfolding of cognate effectors in an ATP hydrolysis-dependent manner'. Similarly, the export gate of the flagellar protein export apparatus is composed of six membrane proteins, FlhA, FlhB, FliO, FliP, FliQ, FliR, and FliI ATPase forms a cytoplasmic hexamer ring complex with FliH and Flij ${ }^{3}$. FliN forms the bottom edge of the $\mathrm{C}$ ring (Fig. 1), which is essential for torque generation and rotational switch of the flagellar motor, to facilitate sequential export of flagellar proteins by providing many FliH binding sites to deliver the FliH-FliI-FliJ-chaperone-substrate complexes to the export gate ${ }^{3,10}$. Thus, the export 


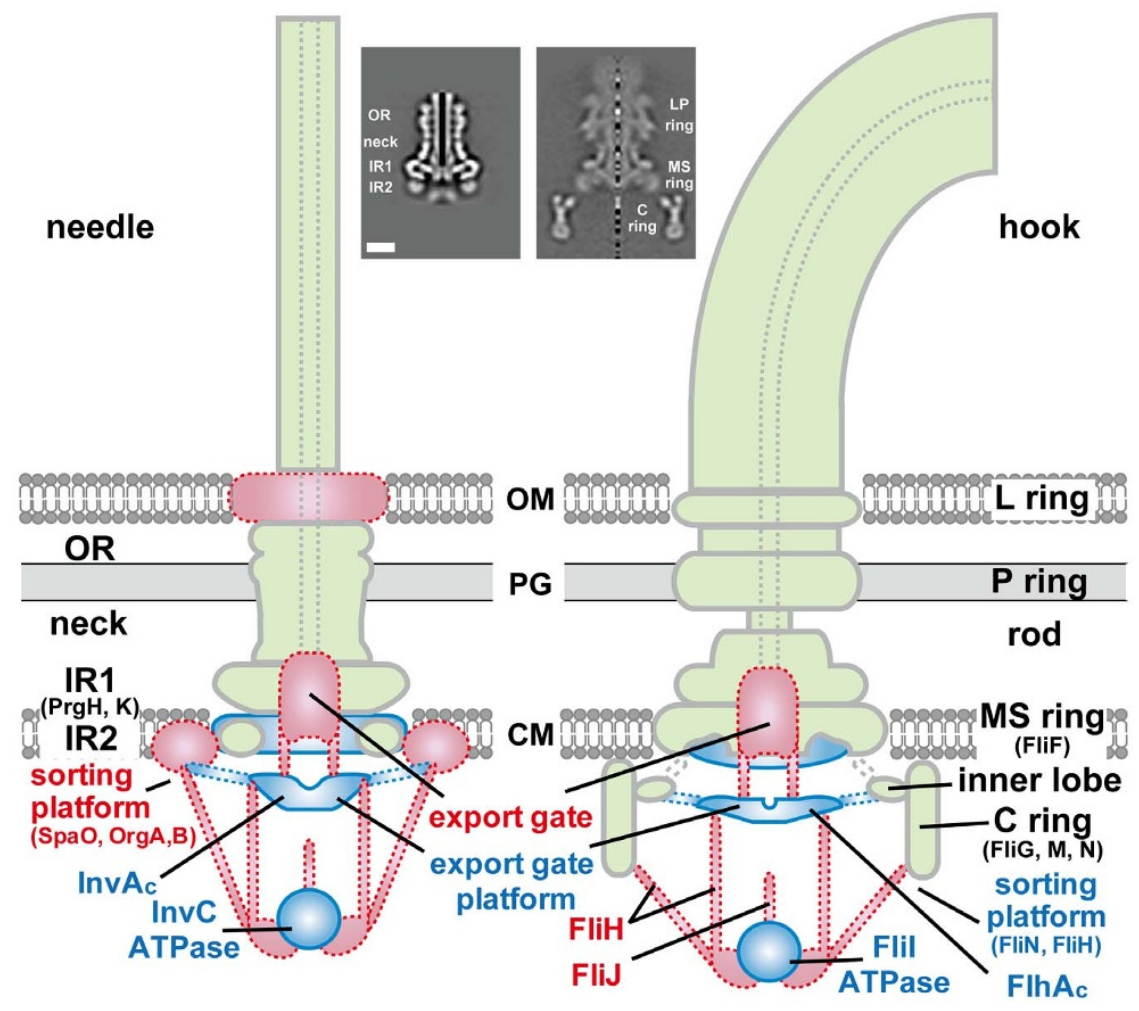

Figure 1 Schematic representation of the structures of Salmonella injectisome (NC, left) and flagellar hook basal body (FBB, right). Different colors indicate structures of different categories: pale green, structures of isolated complexes; blue, additional structures present in the in situ complexes; red, putative structures not yet visible in the in situ complexes. Insets are the axial sections of isolated $\mathrm{NC}^{10}$ and FBB obtained by cryoEM image analysis. OR: outer membrane ring, IR: inner membrane ring, OM: outer membrane, PG: peptidoglycan layer, CM: cytoplasmic membrane.

gate and cytoplasmic structures of FBB play essentially the same roles in protein export as the corresponding two parts of NC, respectively.

The structures of $\mathrm{NC}^{5,11}$ and $\mathrm{FBB}^{6}$ isolated from bacterial cells have been analyzed by electron cryomicroscopy (cryoEM) and single particle image analysis. The central sections of the image reconstructions of $\mathrm{NC}^{5}$ and $\mathrm{FBB}$ are shown in the insets of Fig. 1, and their structures are presented in pale green in the schematic diagram (Fig. 1). Although the structures of the NC IR1 ring made of 24 copies of $\mathrm{PrgH}$ and PrgK and the FBB MS ring made of 26 copies of FliF have been revealed clearly, those of the export gates within these rings are not yet visualized in detail ${ }^{5-7,12}$. The distinct difference between isolated $\mathrm{NC}$ and FBB is the absence of the $\mathrm{C}$ ring in $\mathrm{NC}$ (Fig. 1). That is why precise location of $\mathrm{SpaO}$ is still unknown even though it has been biochemically detected on the cytoplasmic face of $\mathrm{NC}^{13}$. As the NC IR2 ring (Fig. 1) is made of the cytoplasmic domains of $\mathrm{PrgH}$ and $\operatorname{PrgK}^{11}, \mathrm{IR} 2$ is not a candidate for the location of SpaO. Most of all, the structures of isolated $\mathrm{NC}$ and FBB show neither the cytoplasmic domains of the export gate proteins (InvA and SpaS of NC; FlhA and FlhB of FBB), which form another sorting platform for ordered protein export called export gate platform, nor the ATPase complexes (OrgA-OrgB-InvC-secretory protein in NC; FliH-FliI-FliJexport substrate in FBB), which facilitate protein export, probably due to structural disorder or dissociation from NC and FBB by the purification procedure. Additional densities on the cytoplasmic side of isolated NC are highly variable, indicating disorder ${ }^{5,11}$. Therefore, it has been thought that the $\mathrm{C}$ ring of $\mathrm{NC}$, if it exists, may be lost during purification for the same reason. Although in situ structure of Yersinia NC has been revealed recently, the structural details are still obscure $^{14}$. So there are yet no structural clues to the export mechanism. In the present study, we therefore carried out in situ structural analysis of Salmonella NC and FBB by electron cryotomography (ECT).

\section{Results}

Structural analysis of isolated FBB. Although the structure of FBB isolated from Salmonella enterica has already been revealed by cryoEM image analysis ${ }^{6}$, we carried out cryoEM image analysis of FBB isolated from the same strain of Salmonella enterica that we used for in situ structural analysis of $\mathrm{NC}$ and $\mathrm{FBB}$, in order to compare the structures of in situ and isolated FBB as precisely as possible. We isolated and purified FBBs from a wild-type strain of Salmonella with modifications in the genetic background to increase the number of flagella per cell and to form the flagellar basal body without growing the filament and then carried out cryoEM single particle image analysis as described in Methods. The structure is shown in Figs. 1 inset, 4 and 5. We did not find any discernible differences in the structural features from the one previously published ${ }^{6}$.

Structural analysis of in situ NC and FBB. Because the cell body of Salmonella (approximately $1 \mu \mathrm{m}$ ) is too thick even for $300 \mathrm{keV}$ electrons to pass through without too much inelastic and multiple scattering, thereby limiting the resolution of image reconstruction, we constructed mini-cells by overproducing FtsZ (see Methods). The rod shaped cells became nearly spherical with a diameter of $\sim 0.5 \mu \mathrm{m}$ (Fig. 2). Most of the mini-cells had one or two flagella and showed active motility in liquid media (Supplementary movie 1). This assured that FBBs of mini-cells are fully functional, and most likely so are NCs co-present in the mini-cells.

We prepared frozen-hydrated mini-cells on holey carbon grids and carried out ECT to collect tomograms. There were always a few NCs and one or two flagella in the tomogram of each cell (Fig. 3). We often observed NC and FBB side by side, which allowed direct comparison of their in situ structures (Fig. 3b). Both of them showed the structural features that have been revealed for their isolated particles. For FBB, it was possible to recognize the curved 


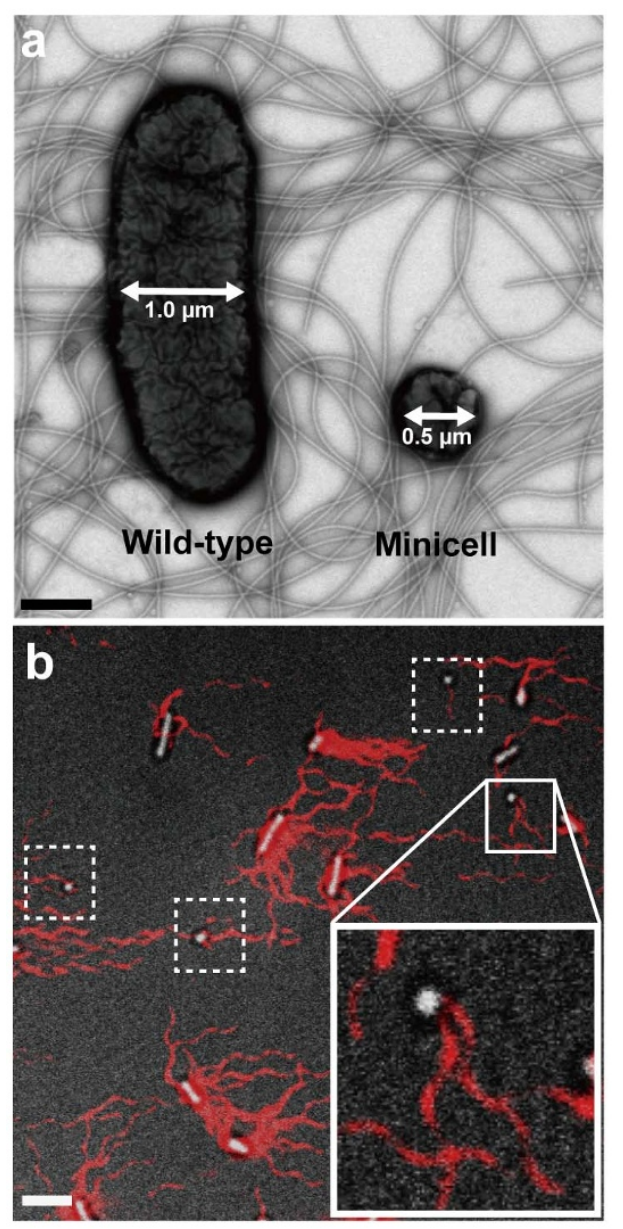

Figure $2 \mid$ Characteristics of Salmonella mini-cell produced by the overproduction of FtsZ. (a) Negatively stained EM image of a Salmonella wild-type cell with a diameter of $\sim 1.0 \mu \mathrm{m}$ and a mini-cell with a diameter of $\sim 0.5 \mu \mathrm{m}$. Scale bar, $500 \mathrm{~nm}$. (b) Bright-field and fluorescence microscopy images of wild-type cells and mini-cells are merged to show cell bodies and the flagellar filaments. The filaments were labeled with the Alexa fluorescent dye (red). Mini-cells are highlighted by square box of dashed or solid line, and the one in the solid-line square is magnified. Scale bar, $4 \mu \mathrm{m}$.

flagellar hook in the cell exterior, the LP ring spanning the outer membrane (OM) and peptidoglycan layer (PG), the MS ring spanning the cytoplasmic membrane (CM), and the $\mathrm{C}$ ring in the cytoplasmic side of the MS ring (see Fig. 1 for each substructure). For NC, the thin needle in the cell exterior and the basal body spanning the periplasmic space (OR, neck and IR1 in Fig. 1) were visible, but no clear structural features were discernible in the cytoplasmic side unlike the $\mathrm{C}$ ring of $\mathrm{FBB}$, although it was predicted from the functional similarity as a sorting platform as discussed below.

We then boxed out subtomograms of NC and FBB and computationally aligned and averaged them to obtain their in situ structures at resolutions high enough to be compared quantitatively with those of isolated NC and FBB obtained by cryoEM single particle image analysis. The number of averaged subtomograms was 75 and 48, and the resolution of image reconstruction was $4.4 \mathrm{~nm}$ and $4.5 \mathrm{~nm}$ for NC and FBB, respectively (Supplementary Fig. 1; Supplementary Table 2 for statistics). Their central sections are shown in Fig. 4.

Structural comparison of in situ and isolated NC and FBB. The structures of NC and FBB in situ both span the outer membrane $(\mathrm{OM})$ and cytoplasmic membranes $(\mathrm{CM})$ in a similar manner
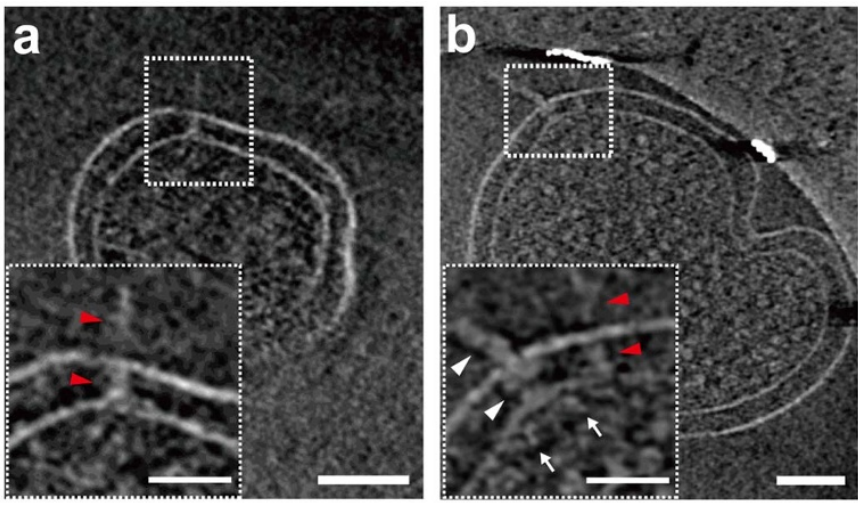

Figure $3 \mid \mathrm{NC}$ and FBB in the tomogram of Salmonella mini-cell. (a) NC and (b) FBB are highlighted with a box on an $11 \mathrm{~nm}$-thick slice of each tomogram. Scale bar, $100 \mathrm{~nm}$. Magnified images of NC and FBB are shown in the insets. The NC and FBB are indicated by red and white arrowheads, respectively. The two small white arrows indicate the $\mathrm{C}$ ring of FBB. Scale bar, $50 \mathrm{~nm}$.

(Fig. 4a, b). The distances between the two membranes are almost the same, and their surface-surface separation forming the periplasmic space is $19 \mathrm{~nm}$. The structures of isolated $\mathrm{NC}^{5}$ and FBB can be well superimposed except for some details, indicating that their core structures are well preserved even after isolation and purification by detergent treatment (Fig. 4c, d). Interestingly, the NC basal body is too short for the OR ring to span the outer membrane (Fig. 4c). Because the shape of isolated NC (OR, neck and IR1) can be superposed over the periplasmic portion of the in situ NC structure relatively well, there may be another ring spanning the outer membrane, rather than elongation of the entire basal body allowing for the OR ring to span the outer membrane as proposed for Yersinia $\mathrm{NC}^{14}$. Since the LP ring is easily detached from isolated FBB in the absence of the hook, suggesting that the hook diameter is large enough to prevent the LP ring from coming off the rod, (Miyata, T., unpublished data) and because the needle, which is attached to the NC OR ring, is also easily detached from the basal body by mechanical perturbation, there may be an unidentified NC ring that spans the outer membrane, and the ring may have been detached from isolated NC as the needle is not thick enough to prevent it.

The densities on the cytoplasmic side, not present in isolated NC and FBB, are also similar to each other, showing a wing-like disc close to CM (red arrow) and a globular density a little farther away (blue arrow). The diameter of the disc and globular densities are $\sim 17 \mathrm{~nm}$ and $\sim 10 \mathrm{~nm}$, respectively. The upper surfaces of the discs are about $3.3 \mathrm{~nm}$ and $4.4 \mathrm{~nm}$ away from the bottom surface of NC and FBB, respectively, and the surface-surface distances between the disc and globular densities are $9.2 \mathrm{~nm}$ and $11 \mathrm{~nm}$, respectively. These two structural features have been reported previously for FBB and assigned to the cytoplasmic domain of FlhA $\left(\right.$ FlhA $\left._{C}\right)$ and the FliI ring complex, respectively ${ }^{15,16}$. The crystal structure of the cytoplasmic domain of Shigella MxiA ( MxiA $\left._{\mathrm{C}}\right)$, a homolog of InvA and FlhA, is a nonameric ring complex (PDB ID 4A5P; ref. 16), and the atomic model can be well fitted into the NC disc density (Fig. 5a, c). A nonameric ring model of Flh $_{C}$ built with the crystal structure of FlhA $_{C}$ (PDB ID 3A5I; ref. 17) was fitted into the FBB disc density as well (Fig. 5b, d). The hexameric ring model of FliI ${ }^{18}$ can also be fitted into the globular densities of NC and FBB (Fig. 5a, b). We therefore identify the two cytoplasmic densities of $\mathrm{NC}$ as the cytoplasmic domain of InvA (InvA $\mathrm{C}_{\mathrm{C}}$ ) forming a nonameric ring and InvC forming a hexameric ring, respectively, although the involvement of the cytoplasmic domains of SpaS and FlhB in the disc density should not be neglected ${ }^{3}$. 

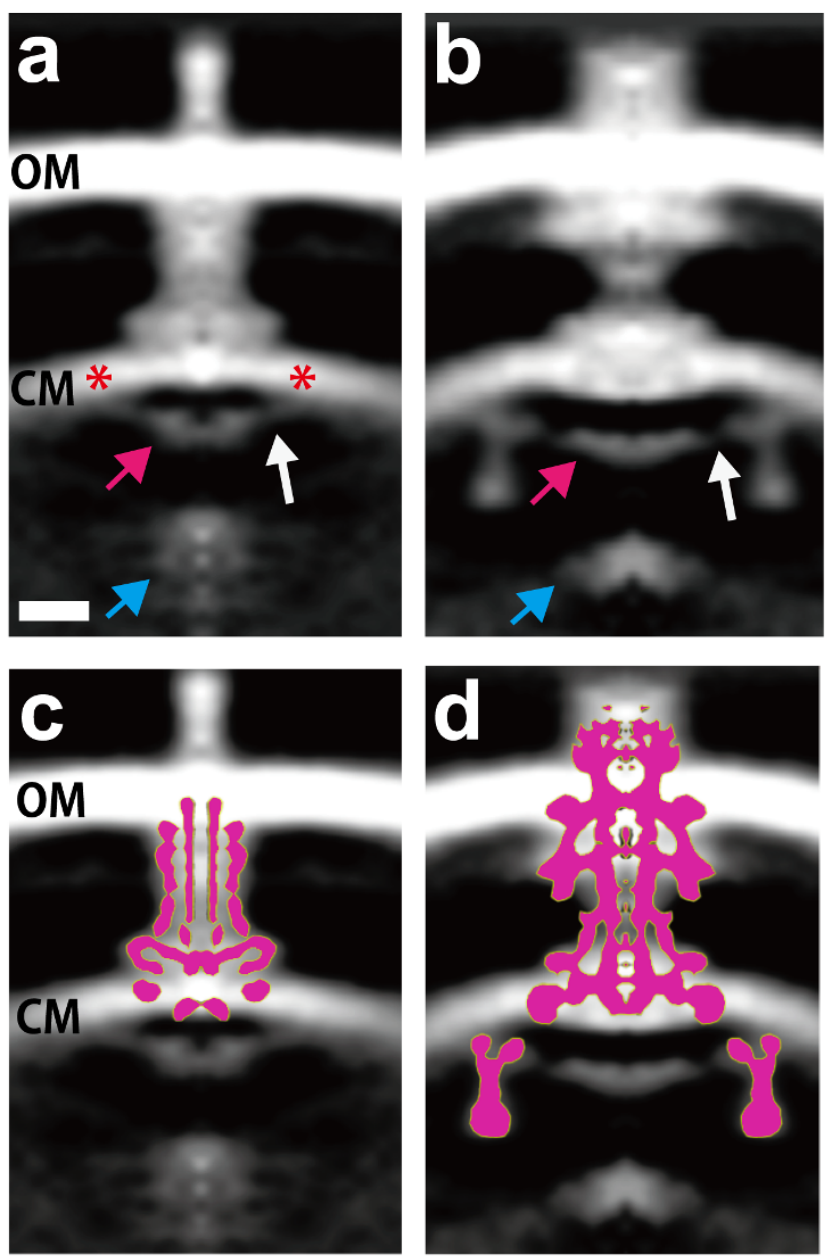

Figure 4 | Comparison of in situ structures of NC and FBB. The central sections of NC (a) and FBB (b) structures in situ after subtomogram average. (c), (d) The axial sections of isolated $\mathrm{NC}^{10}$ and $\mathrm{FBB}$ obtained by cryoEM image analysis are colored yellow and superimposed on their in situ structures, respectively. The cytoplasmic densities designated as the wing-like disc and the globular density in the text are indicated by red and blue arrows and are assigned to InvA $\mathrm{C}_{\mathrm{C}}$ and InvC ATPase in NC and FlhA and FliI ATPase in FBB, respectively. The white arrow indicates the connection between the wing-like disc and a putative structure of the sorting platform composed of $\mathrm{SpaO}, \mathrm{OrgA}$ and $\mathrm{OrgB}$ in $\mathrm{NC}$ and that between the disc and the inner lobe of the $\mathrm{C}$ ring in $\mathrm{FBB}$. The $\mathrm{C}$ ring conformation shows significant differences in the orientations of the cylindrical wall and the inner lobe between the isolated and in situ structures. OM: outer membrane, CM: cytoplasmic membrane. Scale bar, $10 \mathrm{~nm}$.

\section{Discussion}

We now see that NC does not have the density corresponding to the FBB C ring even in situ and that its absence in isolated NC is not due to the isolation procedure. But SpaO must be localized somewhere on the cytoplasmic side of $\mathrm{NC}$ as a sorting platform ${ }^{8}$. What we found as a new structural feature of $\mathrm{NC}$ is a weak but clear density (white arrow) connecting the edge of the wing-like disc to an yet unidentified structural part (asterisk) located on the cytoplasmic surface of the membrane in a radius much farther out $(14 \mathrm{~nm})$ than the outer radii of the IR1 $(12 \mathrm{~nm})$ and IR2 (11 nm) rings (Fig. 4c). This new feature is also present in $\mathrm{FBB}$ except that the connection is made to the inner lobe of the $\mathrm{C}$ ring (Fig. 4b, d). The $\mathrm{C}$ ring and inner lobe show some conformational changes from those of isolated FBB (Fig. 4b, d, 5b), probably due to the extra connection to the disc. In the in situ NC structure, the IR2 ring formed by the cytoplasmic domains of $\mathrm{PrgH}$
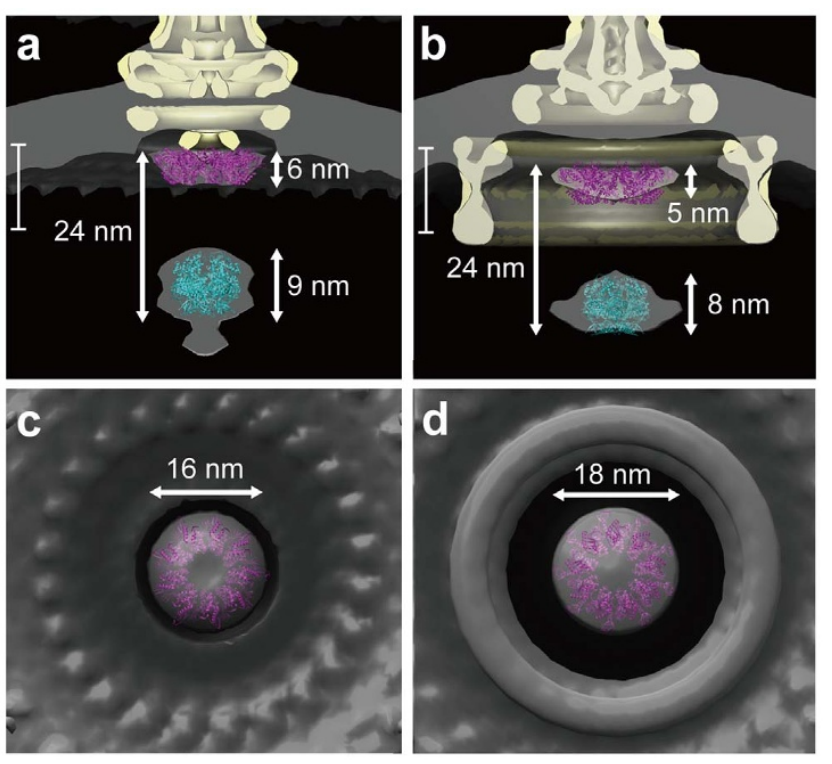

Figure 5 | Superposition of cryoEM density maps of isolated NC and FBB on and docking of the atomic models of $\mathrm{MxiA}_{\mathrm{C}}, \mathrm{FlhA}_{\mathrm{C}}$ and FliI into the density maps of in situ NC and FBB. Magnified views of the membrane and cytoplasmic portions of $\mathrm{NC}(\mathrm{a}, \mathrm{c})$ and $\mathrm{FBB}(\mathrm{b}, \mathrm{d})$. Side views of half-cut section (a,b) and bottom views (c,d) of in situ (grey) and isolated (beige) structures are shown in solid surface representation. The ribbon models in purple are the nonameric rings of Shigella MxiA $_{C}$ (PDB ID 4A5P; ref. 16) $(\mathrm{A}, \mathrm{C})$ and $\mathrm{FlhA}_{\mathrm{C}}{ }^{17}(\mathrm{~b}, \mathrm{~d})$ fitted into the wing-like disc densities, and those in cyan are the hexameric ring model of FliI ATPase ${ }^{18}$ fitted into the globular densities $(\mathrm{a}, \mathrm{b})$.

and $\operatorname{PrgK} \mathrm{K}^{5,11,19}$ is buried in the membrane density, and the extra density observed in some fraction of isolated $\mathrm{NC}$ around the $\mathrm{NC}$ axis below IR2 is not present (Fig. 4a, c), suggesting that the structure of IR2 represents only a portion of the cytoplasmic structure composed of PrgH, PrgK, SpaO, OrgA and OrgB and that the extra density near the NC axis is an artifact produced by the isolation of NC. Considering the important roles of $\mathrm{SpaO}$ and $\mathrm{FliN}$ in facilitating ordered protein export as the sorting platform and the structural features observed for in situ NC and FBB, the cytoplasmic domains of PrgH and PrgK may actually form a ring of larger diameter on the cytoplasmic surface of the membrane, and many copies of SpaO may be bound on the outside of this ring to form another ring of much larger diameter, to which the wing-like disc composed of InvA $\mathrm{C}_{\mathrm{C}}$ is connected as depicted in Fig. 1.

Possible candidates for the connecting densities would be the linker regions of InvA $\left(\operatorname{InvA}_{L}\right)$ and FlhA $\left(\right.$ FlhA $\left._{L}\right)$ connecting their $\mathrm{N}$-terminal transmembrane regions with $\operatorname{InvA}_{C}$ and $\mathrm{FlhA}_{C}$, respectively. But it is unlikely since it would be difficult for these linker chains to be visualized at the present resolution and because the $\mathrm{N}$-terminal transmembrane regions of InvA and FlhA are supposed to be part of the export gate, which is presumably located at the center of the NC IR and FBB MS rings, respectively. FliH may also be a candidate $^{20}$, but its identification requires further experiments. That the connecting densities are visible, albeit weakly, after 24-fold (NC) and 26-fold (FBB) rotational symmetry enforcement suggests that the densities may not represent sparse linkers but actually form a circular sheet that shields against free protein diffusion to the export gate to regulate specific protein export.

The SpaO/YscQ/Spa33 family, which is an essential cytoplasmic component of T3SS, shows a significant sequence similarity to FliN and FliM $^{1}$. The crystal structure of $\mathrm{HscQ}_{\mathrm{B}}$, a SpaO/YscQ/Spa33 ortholog of Pseudomonas syringae, is similar to that of $\mathrm{FliN}^{21,22}$. Immuno-electron microscopy with pull-down assays by GST affinity chromatography has revealed that Shigella Spa33 is localized to the 
NC base through its interactions with MxiG (PrgH) and MxiJ $(\operatorname{PrgK})^{13}$. YscQ is not detected in the NC base of $y s c D(\operatorname{prgH})$ and $y s c J(\operatorname{prgK})$ mutants $^{23}$. These observations further strengthen the possibility that $\mathrm{SpaO}$ associates with the cytoplasmic domains of $\mathrm{PrgH}$ and $\mathrm{PrgK}$ to form a ring as the functional counterpart of the FBB C ring acting as a sorting platform for ordered protein export.

The flagellar protein export apparatus utilizes proton motive force (pmf) across the cytoplasmic membrane ${ }^{24}$. A specific interaction of FliJ with $\mathrm{FlhA}_{\mathrm{L}}$ is brought about by FliH and FliI for efficient, pmfdriven protein export ${ }^{25,26}$. The recruitment of FliH and FliI to the export gate requires the extreme $\mathrm{N}$-terminal region of $\mathrm{FliH}$ that is also responsible for the interaction with $\mathrm{FliN}^{20,27}$. So Fli must be connected with Flh $\mathrm{A}_{\mathrm{C}}$ forming the disc as well as FliN forming the bottom part of the $\mathrm{C}$ ring through FliH (red connections in Fig. 1 right). Likewise, InvC is required for unfolding and export of effector proteins ${ }^{7}$ and must be connected with $\operatorname{Inv}_{\mathrm{C}}$ forming the disc as well as with $\mathrm{SpaO}$ forming a putative cytoplasmic ring through $\mathrm{OrgA}$ and $\mathrm{OrgB}^{8}$ (Fig. 1 left). FliJ and InvI are presumably inserted partially into the central cavities of the FliI and InvC hexamer rings, respectively ${ }^{28}$ (short red bars on ATPases in Fig. 1), so that FliJ and InvI are brought to the vicinity of Flh $A_{C}$ and InvA $A_{C}$ discs for binding to their respective linker regions to convert the export gates into efficient, pmfdriven protein export apparatuses ${ }^{25,26}$. The in situ structures of NC and FBB, however, show that InvC and FliI ATPases are both too far away from the rest (about $10 \mathrm{~nm}$ to the disc) and do not appear to be connected with any part of NC and FBB (Fig. 4, 5). The FliH connections as well as FliJ and InvI are not visible probably because they are all too thin and too few to be visible at the present resolution, especially after imposition of 24- or 26 -fold rotational symmetry. However, the distances are too long for FliJ and InvI to reach Flh $A_{L}$ and InvA $A_{L}$, respectively (Fig. 1), implying that the structures of NC and FBB revealed in this study may represent those of the export-off state. T3SS is activated only upon host cell contact to export effector proteins into the cytosol of host cells in an ordered fashion ${ }^{1,2}$. When the flagellar filaments are broken by viscous shear, the filament regrowth quickly occurs at the distal end of broken filaments ${ }^{29}$. Therefore, we propose that T3SS can turn itself into an off state to save energy as well as proteins and that dynamic conformational changes from what we reported as the export-off state must occur to activate protein export.

\section{Methods}

Culture conditions of mini-cells for ECT. The Salmonella wild-type strain SJW $1103^{30}$ carrying pYVM031, which encodes FtsZ on pBAD24 $4^{31}$, was grown in $5 \mathrm{ml}$ M9 medium (17.1 $\mathrm{g} \mathrm{Na}_{2} \mathrm{HPO}_{4} \cdot 12 \mathrm{H}_{2} \mathrm{O}, 3 \mathrm{~g} \mathrm{KH}_{2} \mathrm{PO}_{4}, 0.5 \mathrm{~g} \mathrm{NaCl}, 1 \mathrm{~g} \mathrm{NH}_{4} \mathrm{Cl}, 0.2 \%$ glycerol, $1 \%$ typtone, $1 \mathrm{mM} \mathrm{MgSO}_{4}$ per litre) containing $50 \mu \mathrm{g} / \mathrm{ml} \mathrm{Ampicillin} \mathrm{at} 30^{\circ} \mathrm{C}$ using $50 \mu \mathrm{l}$ of overnight culture until the culture density reached an $\mathrm{OD}_{600}$ of 0.5 . An arabinose solution was then added to the culture media to a final concentration of $0.2 \%$ for induction of FtsZ overproduction, and the growth continued until the minicell density had reached an $\mathrm{OD}_{600}$ of ca. $1.0^{32}$. After large-size cells and cell debris were removed by centrifugation $\left(4,400 \mathrm{~g}, 5 \mathrm{~min}, 4^{\circ} \mathrm{C}\right)$, the mini-cell fraction was collected by centrifugation $\left(15,300 \mathrm{~g}, 5 \mathrm{~min}, 4^{\circ} \mathrm{C}\right)$. The pellet was resuspended in $50 \mu \mathrm{l}$ of M9 medium.

EM observation of negatively stained samples. Sample solutions were applied to carbon-coated copper grids and negatively stained with $0.5 \%(\mathrm{w} / \mathrm{v})$ phosphotungstic acid. Images were observed with a JEM-1011 transmission electron microscope (JEOL, Tokyo, Japan) operating at $100 \mathrm{kV}$ using a TVIPS TemCam-F415MP CCD camera $(4 \mathrm{k} \times 4 \mathrm{k}, 15 \mu \mathrm{m}$ pixel size).

Fluorescence microscopy. Labeling of flagellar filaments was done as described previously ${ }^{33}$. Salmonella mini-cells were attached to a coverslip (Matsunami glass, Japan), and unattached cells were washed away with motility medium (10 mM potassium phosphate, $0.1 \mathrm{mM}$ EDTA, and $10 \mathrm{mM}$ sodium lactate; $\mathrm{pH}$ 7.0). Polyclonal anti-FliC antibody and anti-rabbit IgG conjugated with Alexa Fluor 594 (Invitrogen) were applied to the cells attached to the coverslip. After washing with motility medium, cells were observed by a an inverted fluorescence microscope (IX71 , Olympus) with a $150 \times$ oil immersion objective lens (UApo150XOTIRFM, NA 1.45, Olympus) and an Electron-Multiplying Charge-Coupled Device (EMCCD) camera (C9100-02, Hamamatsu Photonics).
ECT data collection and tomogram reconstruction. Quantifoil molybdenum 200 mesh R0.6/1.0 grids (Quantifoil Micro Tools) were glow discharged and pretreated with a solution of $10 \mathrm{~nm}$ colloidal gold particles concentrated 1.5 times before use (MP Biomedicals) for tomogram alignment. A $2.6 \mu \mathrm{l}$ sample solution was applied to the grid, blotted by filter paper, and plunged into liquid ethane using Vitrobot (FEI) Images were collected at the liquid-nitrogen temperature using a Titan Krios FEG transmission electron microscope (FEI) operated at $300 \mathrm{kV}$ on FEI Falcon $4 \mathrm{k} \times 4 \mathrm{k}$ direct electron detector (FEI). The magnification was calibrated by measuring the layer-line spacing of $23.0 \AA$ in the Fourier transform of images of tobacco mosaic virus mixed in the sample solution. The pixel size on the specimen was $0.57 \mathrm{~nm}$. Single-axis tilt series were collected covering an angular range from $-70^{\circ}$ to $+70^{\circ}$ with a nonlinear Saxton tilt scheme at 4-7 $\mu \mathrm{m}$ underfocus using the Xplore 3D software package (FEI). A cumulative dose of $200 \mathrm{e}^{-} / \AA^{2}$ or less was used for each tilt series. Images were generally binned two folds and $3 \mathrm{D}$ reconstructions were calculated using the IMOD software package ${ }^{34}$.

Subtomogram averaging. The total number of extracted particles and tomographic reconstructions used in the experiments described in this study are listed in Supplementary Table 2. Individual NCs and FBBs boxed into segments of $82 \times 82 \times$ 82 pixels were manually extracted from the tomographic reconstructions using the IMOD software package ${ }^{34}$. The following processing was performed with the EMAN software package ${ }^{35}$. For coarse alignment, 100 -fold symmetry was applied around the rod axis simply to improve the signal-to-noise ratio of each extracted particles and to eliminate the effect of missing wedge. The 100 -fold symmetry-enforced particles were computationally aligned to a reference with a global 6D (angles and coordinates) cross-correlation scan with an angular step of $8^{\circ}$. The aligned particles were resymmetrized and were then contributed to the reference for the next iteration. After ten iterations, the 24-fold and 26-fold symmetry was applied to the aligned NC and FBB particles, respectively, according to those reported for the NC IR1 ring and FBB MS ring ${ }^{12,36}$. The alignment was refined 5 times with full angle search with $8^{\circ}$ step, 3 times for a local search with $4^{\circ}$ step within the range of $20^{\circ}$ around the highest correlation coefficient angle, 2 times with $2^{\circ}$ step within the range of $10^{\circ}$ around the highest correlation coefficient angle. The coordinates of NC and FBB was stabilized by this iterative refinement. Particles that had correlation coefficients larger than 0.5 with the averaged structure were contributed to the final average. All the processing was performed on a PC cluster computer with 168 CPUs (RC server Calm2000, Real Computing, Tokyo, Japan).

The 3D volumes after subtomogram average were inspected with IMOD software package $^{34}$. The resolution was estimated by FSC of two data sets produced by dividing the particles contributed to the final average into two halves. The 0.5 threshold criterion was used. Solid surface-rending image were obtained by UCSF Chimera ${ }^{37}$. For identification of the known structural features of NC and FBB, the EM maps of $\mathrm{NC}^{5}$, which was deposited in the Electron Microscopy Data Bank under accession number EMDB-1875 and FBB were fitted into the in situ structures of NC and FBB. Figures were arranged with Adobe illustrator (Adobe Systems Inc.).

Purification of FBB. The Salmonella strain SJW2177 $(\Delta f l g K)^{38}$ with deletion of the $c l p P$ gene to increase the number of flagella was used ${ }^{39}$. FBB was isolated as previously described ${ }^{40}$ with some modifications. The cells were grown in $5 \mathrm{~L}$ culture, harvested at late-logarithmic stage, and collected by centrifugation at 4,600 $\mathrm{g}$ for $10 \mathrm{~min}$. The pellets were resuspended in $240 \mathrm{ml}$ of an ice-cold buffer containing $0.5 \mathrm{M}$ sucrose and $50 \mathrm{mM} \mathrm{Tris} / \mathrm{HCl}(\mathrm{pH} 8.0)$. EDTA and lysozyme were added to final concentrations of $10 \mathrm{mM}$ and $0.1 \mathrm{mg} / \mathrm{ml}$, respectively. After the solution was stirred for $30 \mathrm{~min}$, resulting spheroplasts were lysed by adding Triton $\mathrm{X} 100$ and $\mathrm{MgSO}_{4}$ to final concentrations of $1 \%$ and $10 \mathrm{mM}$, respectively, and the solution was stirred again on ice for 1 hour. Unlysed cells and cell debris were removed by centrifugation at $15,000 \mathrm{~g}$ for $20 \mathrm{~min}$, and $\mathrm{pH}$ of the supernatant was adjusted to 10.5 by adding $5 \mathrm{M}$ $\mathrm{NaOH}$. The lysate was spun down by centrifugation at $60,000 \mathrm{~g}$ for $60 \mathrm{~min}$, and the pellet was resuspended in Buffer C (10 mM Tris- $\mathrm{HCl}, 5 \mathrm{mM}$ EDTA, $1 \%$ Triton X100). FBB was collected from a fraction of $20-50 \%$ sucrose density-gradient centrifugation in Buffer C. After centrifugation at $60,000 \mathrm{~g}$ for $60 \mathrm{~min}$, the pellet was resuspended in Buffer S ( $25 \mathrm{mM}$ Tris-HCl pH8.0, 1 mM EDTA, $50 \mathrm{mM} \mathrm{NaCl}, 0.1 \%$ TX100) to remove sucrose.

CryoEM and single particle image analysis of isolated FBB. A $3 \mu \mathrm{FBB}$ solution was applied onto a holey carbon grid (Quantifoil R0.6/1.3, Quantifoil Micro Tools) glowdischarged in a weak vacuum for $5 \mathrm{sec}$ immediately before use. After excess solution was blotted for $3 \sec 2$ times, the grid was quickly frozen by rapidly plunging it into liquid ethane using Vitrobot (FEI). CryoEM images were collected using a JEM3200FSC electron microscope (JEOL) equipped with a liquid-nitrogen cooled specimen stage, an $\Omega$-type energy filter and a field-emission electron gun, operated at an accelerating voltage of $200 \mathrm{kV}$. Images were recorded with a F415mp CCD camera (TVIPS) at a magnification of $88,800 \times$ corresponding to a pixel size of $1.69 \AA$, a defocus range of 1.0-2.5 $\mu \mathrm{m}$ and an electron dose of $40 \mathrm{e}^{-} / \AA^{2}$. Defocus and astigmatism in the image were determined using CTFFIND $3^{41}$. The image processing was carried out using the EMAN software package ${ }^{35}$. FBB images were boxed out with BOXER, aligned, classified and averaged using the REFINE2D.PY program script. Averaged images with out-of-plain tilt angle from $70^{\circ}$ to $90^{\circ}$ were used for $3 \mathrm{D}$ image reconstruction. The refinement of the image analysis was carried out by REFINE with the c100 symmetry. 
Model building and docking into the densities. A nonameric ring model of Flh $\mathrm{A}_{\mathrm{C}}$ was built by fitting the crystal structure of FlhA $_{C}$ (PDB ID 3A5I; ref. 17) to the nonameric ring model of $\mathrm{MxiA}_{\mathrm{C}}$ in the crystal (PDB ID 4A5P; ref. 16) by using UCSF Chimera $^{37}$. The docking of nonameric ring models of $M x i A_{C}$ and $F_{h} A_{C}$ into the disc densities of NC and FBB, respectively, was done also with UCSF Chimera ${ }^{37}$

1. Hueck, C. J. Type III protein secretion systems in bacterial pathogens of animals and plants. Mol. Biol. Rev. 62, 379-433 (1998).

2. Kosarewicz, A., Königsmaier, L. \& Marlovits, T. C. The blueprint of the type-3 injectisome. Philos. Trans. R. Soc, Lond. B Biol. Sci. 367, 1140-1154 (2012).

3. Minamino, T., Imada, K. \& Namba, K. Mechanisms of type III protein export for bacterial flagellar assembly. Mol. Biosyst. 4, 1105-1115 (2008).

4. Kubori, T. et al. Supramolecular structure of the Salmonella typhimurium type III protein secretion system. Science. 280, 602-605 (1998).

5. Schraidt, O. \& Marlovits, T. C. Three-dimensional model of Salmonella's needle complex at subnanometer resolution. Science. 331, 1192-1195 (2011).

6. Thomas, D. R., Francis, N. R., Xu, C. \& DeRosier, D. J. The three-dimensional structure of the flagellar rotor from a clockwise-locked mutant of Salmonella enterica serovar Typhimurium. J. Bacteriol. 188, 7039-7048 (2006).

7. Wagner, S. et al. Organization and coordinated assembly of the type III secretion export apparatus. Proc. Natl. Acad. Sci. U.S.A. 107, 17745-17750 (2010).

8. Lara-Tejero, M., Kato, J., Wagner, S., Liu, X. \& Galán, J. E. A sorting platform determines the order of protein secretion in bacterial type III systems. Science. 331, 1188-1191 (2011)

9. Akeda, Y. \& Galán, J. E. Chaperone release and unfolding of substrates in type III secretion. Nature. 437, 911-915 (2005).

10. González-Pedrajo, B., Minamino, T., Kihara, M. \& Namba, K. Interactions between $\mathrm{C}$ ring proteins and export apparatus components: a possible mechanism for facilitating type III protein export. Mol. Micriobiol. 60, 984-998 (2006).

11. Schraidt, O. et al. Topology and organization of the Salmonella typhimurium type III secretion needle complex components. PLoS Pathog. 6, e1000824 (2010).

12. Suzuki, H., Yonekura, K. \& Namba, K. Structure of the rotor of the bacterial flagellar motor revealed by electron cryomicroscopy and single-particle image analysis. J. Mol. Biol. 337, 105-113 (2004).

13. Morita-Ishihara, T. et al. Shigella Spa33 is an essential C-ring component of type III secretion machinery. J. Biol. Chem. 281, 599-607 (2006).

14. Kudryashev, M. et al. In situ structural analysis of the Yersinia enterocolitica injectisome. eLife. 2, e00792 (2013).

15. Chen, S. et al. Structure diversity of bacterial flagellar motors. EMBO J. $\mathbf{3 0}$, 2972-2981 (2011).

16. Abrusci, P. et al. Architecture of the major component of the type III secretion system export apparatus. Nat. Struct. Mol. Biol. 20, 99-104 (2012).

17. Saijo-Hamano, Y. et al. Structure of the cytoplasmic domain of FlhA and implication for flagellar type III protein export. Mol. Microbiol. 76, 260-268 (2010)

18. Imada, K., Minamino, T., Tahara, A. \& Namba, K. Structure similarity between the flagellar type III ATPase FliI and F1-ATPase subunits. Proc. Natl. Acad. Sci. U.S.A. 104, 485-490 (2007).

19. Bergeron, J. R. C. et al. A refined model of the prototypical Salmonella SPI-1 T3SS basal body reveals the molecular basis for its assembly. PLOS Pathog. 9, e1003307 (2013).

20. Minamino, T. et al. Roles of the extreme N-terminal region of FliH for efficient localization of the FliH-FliI complex to the bacterial flagellar type III export apparatus. Mol. Microbiol. 74, 1471-1483 (2009).

21. Fadouloglou, V. E. et al. Structure of $\mathrm{HrcQ}_{B}-\mathrm{C}$, a conserved component of the bacterial type III secretion systems. Proc. Natl. Acad. Sci. U.S.A. 101, 70-75 (2004).

22. Brown, P. N., Mathews, M. A. A., Joss, L. A., Hill, C. P. \& Blair, D. F. Crystal structure of the flagellar rotor protein FliN from Thermotoga maritima. J. Bacteriol. 187, 2890 (2005).

23. Diepold, A. et al. Deciphering the assembly of the Yersinia type III secretion injectisome. EMBO J. 29, 1928-1940 (2010).

24. Minamino, T. \& Namba, K. Distinct roles of the FliI ATPase and proton motive force in bacterial flagellar protein export. Nature. 451, 485-488 (2008).

25. Minamino, T., Morimoto, Y. V., Hara, N. \& Namba, K. An energy transduction mechanism used in bacterial flagellar type III protein export. Nat. Commun. 2, 475 (2011).

26. Ibuki, T. et al. Interaction between FliJ and FlhA, components of the bacterial flagellar type III export apparatus. J. Bacteriol. 195, 466-473 (2013).

27. Hara, N., Morimoto, Y. V., Kawamoto, A., Namba, K. \& Minamino, T. Interaction of the extreme $\mathrm{N}$-terminal region of $\mathrm{FliH}$ with FlhA is required for efficient bacterial flagellar protein export. J. Bacteriol. 194, 5353-5360 (2012).
28. Ibuki, T. et al. Common architecture of the flagellar type III protein export apparatus and F- and V-type ATPases. Nat. Struct. Mol. Biol. 18, 277-282 (2011).

29. Rosu, V. \& Hughes, K. T. sigma28-dependent transcription in Salmonella enterica is independent of flagellar shearing. J. Bacteriol. 188, 5196 (2006).

30. Yamaguchi, S., Fujita, H., Ishihara, A., Aizawa, S. \& Macnab, R. M. Subdivision of flagellar genes of Salmonella typhimurium into regions responsible for assembly, rotation, and switching. J. Bacteriol. 166, 187-193 (1986).

31. Guzman, L. M., Belin, D., Carson, M. J. \& Beckwith, J. Tight regulation, modulation, and high-level expression by vectors containing the arabinose PBAD promoter. J. Bacteriol. 177, 4121-4130 (1995).

32. Briegel, A. et al. Bacterial chemoreceptor arrays are hexagonally packed trimmers of receptor dimers networked by rings of kinase and coupling proteins. Proc Natl Acad Sci USA. 109, 3766-3771 (2012)

33. Morimoto, V. Y., Nakamura, S., Kami-ike, N., Namba, K. \& Minamino, T. Charged residues in the cytoplasmic loop of MotA are required for stator assembly into the bacterial flagellar motor. Mol. Microbiol. 78, 1117-1129 (2010).

34. Kremer, J. R., Mastronarde, D. N. \& McIntosh, J. R. Computer visualization of three-dimensional image data using IMOD. J. Struct Biol. 116, 71-76 (1996).

35. Ludtke, S. J., Baldwin, P. R. \& Chiu, W. EMAN: semiautomated software for highresolution single particle reconstructions. J. Struct. Biol. 128, 82-97 (1999).

36. Yip, C. K. et al. Structural characterization of the molecular platform for type III secretion system assembly. Nature 435, 702-707 (2005).

37. Pettersen, E. F. et al. UCSF Chimera-a visualization system for exploratory research and analysis. J. Comput. Chem. 25, 1605-1612 (2004).

38. Homma, M., Kutsukake, K., Iino, T. \& Yamaguchi, S. Hook-associated proteins essential for flagellar filament formation in Salmonella typhimurium. J. Bacteriol. 157, 100108 (1984)

39. Tomoyasu, T. et al. The ClpXP ATP-dependent protease regulates flagellum synthesis in Salmonella entrica serovar typhimurium. J. Bacteriol. 184, 645-653 (2002).

40. Francis, N. R., Sosinsky, G. E., Thomas, D. \& DeRosier, D. J. Isolation, characterization and structure of bacterial flagellar motors containing the switch complex. J. Mol. Biol. 235, 1261-1270 (1994).

41. Mindell, J. A. \& Grigorieff, N. Accurate determination of local defocus and specimen tilt in electron microscopy. J. Struct. Biol. 142, 334-347 (2003).

\section{Acknowledgments}

We thank Tomoko Kubori for fruitful discussion and comments on the results and Hideyuki Matsunami for a Salmonella mutant strain to isolate FBB. A.K. and Y.V.M. were research fellows of the Japan Society for the Promotion of Science. This work was supported in part by Grants-in-Aid for Scientific Research (21227006 and 25000013 to K.N. 22570161,2312516 and 23115008 to T.Minamino; 24570131 to T.Miyata.; and 24117001a and 24117004 a to T.K.).

\section{Author contributions}

A.K. prepared specimens and carried out ECT data collection and analysis, for which T.K. gave help and guidance. Y.V.M. and K.T.H. made constructs for minicell production. T.Miyata and T.K. carried out cryoEM single particle image analysis of isolated FBB. K.N planed the whole project and supervised the project. A.K., T.Minamino and K.N. wrote the paper based on discussion with other authors, and K.T.H. did English proofreading.

\section{Additional information}

Supplementary information accompanies this paper at http://www.nature.com/ scientificreports

Data deposition: The density maps of NC and FBB have been deposited in the Electron Microscopy Data Bank, http://www.emdatabank.org/ (accession code: EMD-2520 and EMD-2521).

Competing financial interests: The authors declare no competing financial interests

How to cite this article: Kawamoto, A. et al. Common and distinct structural features of Salmonella injectisome and flagellar basal body. Sci. Rep. 3, 3369; DOI:10.1038/srep03369 (2013)

(c) (i) $\Theta \Theta$ This work is licensed under a Creative Commons Attribution-

By nc No NonCommercial-NoDerivs 3.0 Unported license. To view a copy of this license, visit http://creativecommons.org/licenses/by-nc-nd/3.0 